\title{
CpG site hypomethylation at ETS1-binding region regulates DLK1 expression in Chinese patients with Tetralogy of Fallot
}

\author{
GUIXIANG TIAN ${ }^{1-3^{*}}$, LILI HE ${ }^{1,3^{*}}$, RUOYI GU ${ }^{1-3^{*}}$, JINGWEI SUN $^{4}$, WEICHENG CHEN $^{1,3}$, \\ YANYAN QIAN $^{1-3}$, XIAOJING MA $^{1,3}$, WEILI YAN ${ }^{1-3}$, ZHENSHAN ZHAO $^{1-3}$, ZIQING XU $^{1-3}$, \\ MEIJIAO SUO ${ }^{1-3}$, WEI SHENG ${ }^{1-3}$ and GUOYING HUANG ${ }^{1-3}$ \\ ${ }^{1}$ Department of Ultrasound, Cardiovascular Center, Pediatrics Research Institute, National Children's Medical Center, \\ Children's Hospital of Fudan University; ${ }^{2}$ Shanghai Key Laboratory of Birth Defects, Children's Hospital of Fudan University; \\ ${ }^{3}$ Research Unit of Early Intervention of Genetically Related Childhood Cardiovascular Diseases, \\ Chinese Academy of Medical Sciences, Shanghai 201102; ${ }^{4}$ Pediatrics Department, \\ Bengbu First People's Hospital, Bengbu, Anhui 233000, P.R. China
}

Received July 22, 2021; Accepted November 29, 2021

DOI: $10.3892 / \mathrm{mmr} .2022 .12609$

\begin{abstract}
Tetralogy of Fallot (TOF) is the most common cyanotic congenital heart malformation accounting for $\sim 10 \%$ of cases. Although the pathogenesis of TOF is complex and largely unknown, epigenetics plays a huge role, specifically DNA methylation. The protein $\delta$ like non-canonical Notch ligand 1 (DLK1) gene encodes a non-canonical ligand of the Notch signaling pathway, which is involved in heart development. However, the epigenetic mechanism of DLK1 in the pathogenesis of TOF is yet to be elucidated. Therefore, the present study aimed to clarify its specific mechanism. In this study, immunohistochemistry was used to detect the protein expression of DLK1 and the methylation status of the DLK1 promoter was measured via bisulfite sequencing PCR. Dual-luciferase reporter assays were performed to examine the influence of transcription factor ETS proto-oncogene 1 (ETS1) on DLK1 gene expression. The electrophoretic mobility shift assay and chromatin immunoprecipitation assay, both in vivo and in vitro, were used to verify the binding of the ETS1 transcription factor to the DLK1 promoter as well as the influence of methylation status of DLK1 promoter on this binding affinity. The expression of DLK1 in the right
\end{abstract}

Correspondence to: Professor Wei Sheng or Professor Guoying Huang, Department of Ultrasound, Cardiovascular Center, Pediatrics Research Institute, National Children's Medical Center, Children's Hospital of Fudan University, 399 Wanyuan Road, Shanghai 201102, P.R. China

E-mail: sheng4616@126.com

E-mail: gyhuang@shmu.edu.cn

${ }^{*}$ Contributed equally

Key words: $\delta$ like non-canonical Notch ligand 1, DNA methylation, ETS proto-oncogene 1, Tetralogy of Fallot ventricular outflow tract was significantly lower in patients with Tetralogy of Fallot (TOF) than that in controls $(\mathrm{P}<0.001)$. Moreover, the methylation level of $\mathrm{CpG}$ site 10 and $\mathrm{CpG}$ site 11 in the DLK1_R region was significantly decreased in TOF cases compared with controls $(\mathrm{P}<0.01)$. The integral methylation levels of DLK1_R and the methylation status of the $\mathrm{CpG}$ site 11 were both positively associated with DLK1 protein expression in TOF cases. ETS1 was found to inhibit DLK1 transcriptional activity by binding to the CpG site 11 and this affinity could be influenced by the methylation level of the DLK1 promoter. These findings demonstrated that the hypomethylation of the DLK1 promoter could increase the binding affinity of ETS1 transcription factor, which in turn inhibited DLK1 gene transcriptional activity and contributed to the development of TOF.

\section{Introduction}

Tetralogy of Fallot (TOF) is the most common cyanotic congenital heart disease with a prevalence of 1/3,600 live births (1). Its symptoms consist of pulmonary outflow tract obstruction, ventricular septal defects (VSDs), overriding aortic roots and right ventricular hypertrophy (2). Although advances in pharmacotherapy and surgical procedures have improved the survival rate of patients with TOF, the exact pathogenesis of TOF is yet to be elucidated. However, only $20 \%$ of total TOF cases have a known cause, which may be associated with gene mutations or chromosomal anomalies, thus the exact etiology for the remaining TOF cases remains unclear (2). In addition to genetic mechanisms, epigenetics may play an important role in the development of TOF (3).

The Notch signaling pathway is highly conserved and related to the formation of the atrioventricular ducts, valves, outflow tracts and trabecula $(4,5)$. Mutations in the genes of the Notch signaling pathway can cause heart defects in humans or mice, proving its important role in cardiac development (6-8). $\delta$ like non-canonical Notch ligand 1 (DLK1) is a non-canonical Notch ligand that regulates the Notch signaling pathway (9). 
Several studies have found that DLK1 is involved in the cell differentiation process throughout embryonic development and adulthood (9). During cardiac development, a previous study demonstrated that DLK1 is highly expressed in the heart of embryos (10). In addition, DLK1 can negatively regulate the differentiation of cardiac fibroblasts into myofibroblasts, and therefore control myocardial fibrosis (11). Furthermore, a previous study reported that mutations of zinc finger protein 57 homolog, the target gene of DLK1, can cause a variety of cardiac defects, including atrial septal defect and VSD, via the Notch signaling pathway (10). Moreover, Page et al (2) found that there was a very low frequency of genetic variants in the DLK1 coding region in 829 patients with TOF (2). Therefore, these previous studies indicate that epigenetic changes of the DLK1 gene may be a risk factor for the pathogenesis of TOF.

DNA methylation is the most thoroughly studied epigenetic regulatory mechanism and can alter gene expression in both development and disease (12). Numerous studies have shown that aberrant DNA methylation may be associated with cardiovascular disease (13-15). For example, abnormal DNA methylation of several candidate genes involved in cardiac development, such as transcription factor GATA-4, has been found in patients with congenital heart disease (CHD) (3). In addition, aberrant methylation levels of the homeobox protein Nkx-2.5 gene body and heart and neural crest derivatives-expressed protein 1 gene promoter region are negatively correlated with their mRNA expression (16). In the present study, the changes in DNA methylation of the DLK1 promoter region in TOF were explored and its influence on gene expression was analyzed. These findings may provide important clues in understanding the etiology of this disease.

\section{Materials and methods}

Clinical tissue samples. The Ethics Committee of the Children's Hospital of Fudan University [Shanghai, China; approval no. 2015(26)] and Soochow University (Suzhou, China) approved the collection of cardiac tissues. Written informed consent of all study participants was obtained from their parents or relatives, and the study was registered in the Chinese Clinical Trial Registry (registration no. ChiCTR2100051811). TOF was diagnosed in 25 patients recruited from the Children's Hospital of Fudan University between January 2016 and July 2018. They were diagnosed by echocardiogram and confirmed by surgery. The five control samples obtained from the Department of Forensic Medicine of Soochow University were patients who had passed away from accidents without any known heart problems. The clinical characteristics of the samples are summarized in Table I.

Immunohistochemistry. The right ventricular outflow tract (RVOT) tissues of patients with TOF and controls were fixed with $10 \%$ neutral buffered formalin for $\sim 48 \mathrm{~h}$ at room temperature. The fixed tissues were embedded in paraffin and cut into $4 \mu \mathrm{m}$ thick sections. The paraffin-embedded sections were baked in $56^{\circ} \mathrm{C}$ for $\sim 3 \mathrm{~h}$, dewaxed in dimethylbenzene, hydrated in descending alcohol series (100\%, $5 \mathrm{~min} ; 100 \%, 5 \mathrm{~min}$; $\sim 95 \%, 5 \mathrm{~min}$; 85\%, $5 \mathrm{~min} ; \sim 75 \%, 5 \mathrm{~min})$ and boiled $\left(100^{\circ} \mathrm{C}\right)$ for antigen retrieval with citrate buffer $(0.01 \mathrm{~mol} / \mathrm{l} ; \mathrm{pH}, 6.0)$ (Beyotime Institute of Biotechnology). Next, endogenous
Table I. Demographic characteristics of TOF cases $(n=25)$ and controls $(n=5)$.

\begin{tabular}{lcc}
\hline Characteristics & TOF & Control \\
\hline $\begin{array}{l}\text { Age, years, median } \\
(\text { IQR) }\end{array}$ & $0.59(0.37-0.95)$ & $0.17(0.01-0.79)$ \\
$<1, \mathrm{n}(\%)$ & $20(80)$ & $4(80)$ \\
$1 \sim 2, \mathrm{n}(\%)$ & $4(16)$ & $1(20)$ \\
$>2, \mathrm{n}(\%)$ & $1(4)$ & $0(0)$ \\
Sex & & \\
Female, $\mathrm{n}(\%)$ & $10(40)$ & $0(0)$ \\
Male, n $(\%)$ & $15(60)$ & $5(100)$ \\
\hline
\end{tabular}

TOF, Tetralogy of Fallot.

peroxidase activity was blocked with $3 \%$ hydrogen peroxide for $\sim 30 \mathrm{~min}$ at room temperature, and $5 \%$ bovine serum $(0.05 \mathrm{~g} / \mathrm{ml}$; BioFroxx; neoFroxx $\mathrm{GmbH}$ ) was used to reduce non-specific staining. The sections were incubated with a primary antibody against DLK1 (1:200; cat. no. ab210471; Abcam) overnight at $4^{\circ} \mathrm{C}$, followed by incubation with horseradish peroxidase (HRP)-conjugated anti-rabbit/anti-mouse IgG antibodies (cat. no. GK500710; Gene Tech Co., Ltd.) for $2 \mathrm{~h}$ at $25^{\circ} \mathrm{C}$. Lastly, DAB and hematoxylin were applied for staining. The intensity of DLK1 protein expression was detected under a light microscope (Lecia Microsystems GmbH). For each sample, three fields of view without repeating areas were selected under the light microscope (magnification, x200) and images were captured in order to analyze the expression of DLK1 in RVOT tissues using ImageJ software version 1.48 (National Institutes of Health). Segmentation was set at a level that allowed for the detection of positive immunostaining and the positive area $\%$ of each image was measured. The mean value of the total of three visual fields was calculated.

DNA extraction and bisulfite sequencing PCR (BSP). Genomic DNA from the RVOT tissues of controls and patients with TOF was extracted using the E.Z.N.A. ${ }^{\circledR}$ Tissue DNA Kit (cat. no. D3396; Omega Bio-Tek, Inc.) following the manufacturer's instructions. Bisulfite modification of genomic DNA was carried out using the EZ DNA Methylation-Gold kit (Zymo Research Corp.) and amplified by PCR with ZymoTaq PreMix (Zymo Research Corp.). The primers were designed using Methyl Primer Express ${ }^{\mathrm{TM}}$ v1.0 software (Applied Biosystem; Thermo Fisher Scientific, Inc.): DLK1-BSP-forward (F), 5'-TAGTTGGGTATGTGTGTTTGTG-3' and reverse (R), 5'-TTACCCAACCATAAACATCCT-3'. Thermocycling conditions were as follows: $95^{\circ} \mathrm{C}$ for $5 \mathrm{~min} ; 40$ cycles of $95^{\circ} \mathrm{C}$ for $30 \mathrm{sec}, 58^{\circ} \mathrm{C}$ for $30 \mathrm{sec}$ and $72^{\circ} \mathrm{C}$ for $40 \mathrm{sec}$; and $72^{\circ} \mathrm{C}$ for $7 \mathrm{~min}$.

The PCR products were purified (Axygen; Corning, Inc.), inserted into a pGEM-T easy vector (Promega Corporation), and then transformed into $E$. coli DH5 $\alpha$ competent cells (cat. no. DL1002; Shanghai Weidi Biotechnology Co., Ltd.). A total of 10 clones were selected randomly to determine the methylation status. The sequencing results were analyzed using the QUMA website (http://quma.cdb.riken.jp). 
Table II. Sequences of oligonucleotide probes used for electrophoretic mobility shift assay.

Probe Sequences $\left(5^{\prime} \rightarrow 3^{\prime}\right)$

Biotin-DLK1_-744/-720

F: TCTGTTTATACGTGTGTTTGCGTGT

Unlabeled-DLK1_-744/-720

R: ACACGCAAACACACGTATAAACAGA

F: TCTGTTTATACGTGTGTTTGCGTGT

R: ACACGCAAACACACGTATAAACAGA

MU-DLK1_-744/-720

F: TCTGTTTATACGTGTGGCGATATGT

R: ACATATCGCCACACGTATAAACAGA

Me-DLK1_-744/-720

F: TCTGTTTATACGTGTGTTTGCGTGT

R: ACACGCAAACACACGTATAAACAGA

DLK1, $\delta$ like non-canonical Notch ligand 1; Me, methylated; MU, mutant; F, forward; R, reverse.

Luciferase reporter plasmid construction. pGL3-DLK1_ $-902 /-597$ was constructed by amplifying the fragment containing DLK1-R (-899 to $-641 \mathrm{bp})$ using the following primers: DLK1-KpnI-F, 5'-ATAGGTACCAGTCAGCTGGGT ATGTGTGC-3'; DLK1-XhoI-R, 5'-GATCTCGAGTATAGA CACAGTCCTAGGTGGCAG-3'. Next, the amplified fragment was inserted into the pGL3-Promoter (Promega Corporation) vector to determine the influence of the DLK1_R region on gene transcriptional activity.

Cell culture, transfection and dual-luciferase reporter gene assay. 293 cells (The Cell Bank of Type Culture Collection of The Chinese Academy of Sciences) were plated in 96-well plates at $2-4 \times 10^{5}$ cells/well and grown in DMEM (Gibco; Thermo Fisher Scientific, Inc). containing 10\% FBS (Gibco; Thermo Fisher Scientific, Inc.) and penicillin-streptomycin (1:100; Gibco, Thermo Fisher Scientific, Inc.) at $37^{\circ} \mathrm{C}$ under $5 \%$ $\mathrm{CO}_{2}$. Once cells reached $60 \%$ confluency, $100 \mathrm{ng}$ pGL3-basic, pGL3-promoter and pGL3-DLK1_-902/-597 were separately co-transfected with $4 \mathrm{ng}$ internal reference pRL-TK plasmids (Promega Corporation) using Lipofectamine ${ }^{\circledR} 3000$ (Invitrogen; Thermo Fisher Scientific, Inc.) at $25^{\circ} \mathrm{C}$. The transfection process lasted 30-60 min.

The JASPAR database (version JASPAR 2020) was used to analyze the sequence of the DLK1_R region and binding sites for the protein C-ets-1 (ETS1) transcription factor containing the $\mathrm{CpG}$ site 11 were found (17). Therefore, $100 \mathrm{ng}$ pcDNA3.1-ETS1 and $100 \mathrm{ng}$ constructed plasmids were co-transfected into cells. Luciferase activity was detected using the dual-luciferase reporter assay system (Promega Corporation) $48 \mathrm{~h}$ after transfection. Three independent luciferase activity assays were conducted. PGL3-basic vector served as a negative control, and the pGL3-promoter vector was a positive control. PRL-TK plasmids were used to normalize the luciferase activity to avoid errors caused by differences in the number of cell and efficiency of plasmid transfection to cells. To compare the differences between the two groups, data of three times experiments were normalized relative to the pGL3-promoter group.

Electrophoretic mobility shift assay (EMSA) and Shift-western blotting. 293 cells were plated in $10 \mathrm{~cm}$ dishes at $2 \times 10^{6}$ cells/well. Once cells reached $70 \%$ confluency, $12.5 \mu \mathrm{g}$
pcDNA3.1 and pcDNA3.1-ETS1 were separately transfected into cells using Lipofectamine 3000 at $25^{\circ} \mathrm{C}$ according to the manufacturer's protocol. The transfection process lasted 30-60 min. The pcDNA3.1 plasmid (the empty vector) was used as the negative control. After $48 \mathrm{~h}$, the nuclear protein was extracted by using cytoplasmic extraction reagent and nuclear extraction reagent (Thermo Fisher Scientific, Inc.), and the concentration of the nuclear protein was measured using BCA protein assay (Takara Biotechnology Co., Ltd.) according to the manufacturer's protocol. Furthermore, western blotting was performed to verify the overexpression of ETS1. Samples containing equal amounts of protein $(15 \mu \mathrm{g})$ were separated by SDS-PAGE on $10 \%$ gels (Epizyme, Inc.), and subsequently transferred to nitrocellulose membranes (MilliporeSigma) and blocked with $5 \%$ skimmed milk $(0.05 \mathrm{~g} / \mathrm{ml})$ for $\sim 1 \mathrm{~h}$ at room temperature. The membranes were probed with primary antibodies against ETS1 (1:1,000; cat. no. D8O8A; Cell Signaling Technology, Inc.) and PCNA (1:5,000; cat. no. 10205-2-AP; ProteinTech Group, Inc.) at $4^{\circ} \mathrm{C}$ overnight. Then, membranes were incubated with HRP-conjugated anti-rabbit secondary antibody (1:5,000; cat. no. M21002; Abmart Pharmaceutical Technology Co., Ltd.) for $\sim 2 \mathrm{~h}$ at room temperature. The blots were visualized using enhanced chemiluminescence reagents (Thermo Fisher Scientific, Inc.).

Biotin-labeled oligonucleotide probes containing the binding sequence of ETS1 were synthesized and labeled with biotin at the 5 ' end. Unlabeled probes used for the competition were also synthesized and the methylated probes were modified at $\mathrm{CpG}$ sites. These probes were purchased from Generay Biotech Co., Ltd. The sequences of the DNA probes are shown in Table II. ETS1 protein $(10 \mu \mathrm{g})$ was co-incubated with 4 pmol unlabeled probes (1:100 dilution) for $\sim 30 \mathrm{~min}$ at room temperature and subsequently with 20 fmol biotin-labeled probes (1:10,000 dilution) for $\sim 30 \mathrm{~min}$ at room temperature. The protein-DNA complexes were separated from free DNA probes on a $6 \%$ polyacrylamide gel in $0.5 \mathrm{X}$ TBE at $100 \mathrm{~V}$ for $\sim 50 \mathrm{~min}$ and then transferred to a nylon membrane (MilliporeSigma). Subsequently, the membrane was detected with the LightShift ${ }^{\mathrm{TM}}$ EMSA kit (Thermo Fisher Scientific, Inc.) according to the manufacturer's protocol. The Shift-western blotting was performed using the same methods as EMSA, except that EMSA involved the use of nylon membranes, while Shift-western blotting required nitrocellulose membranes for 
A

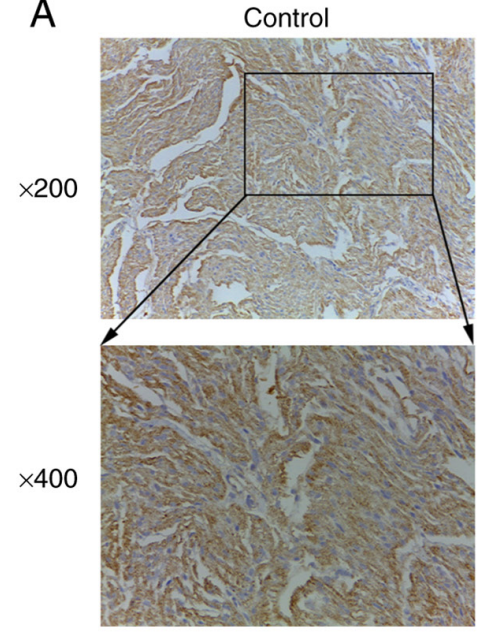

TOF

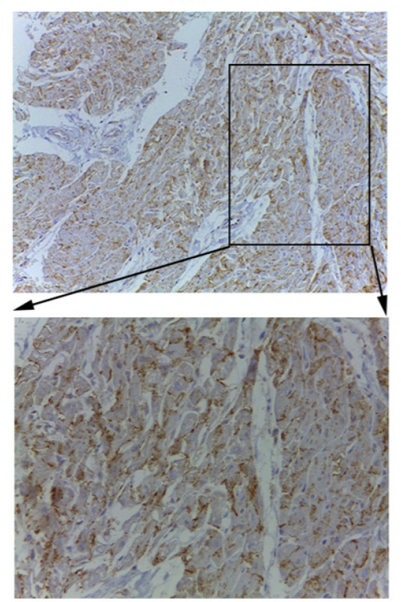

B

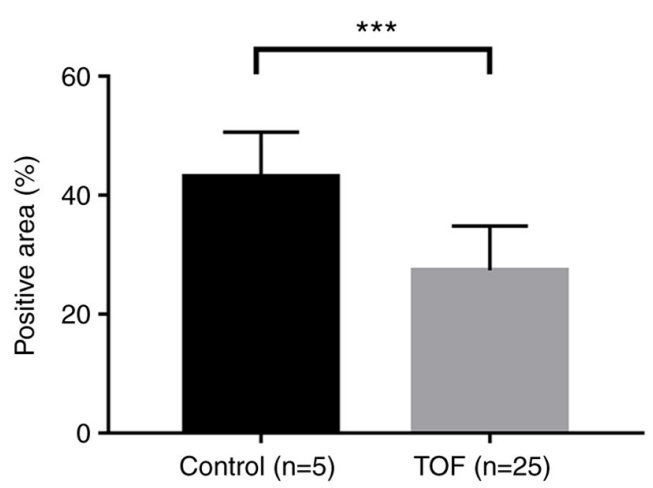

Figure 1. Expression analysis of DLK1 protein in cardiac tissues. (A) DLK1 protein expression was detected by immunohistochemistry [anti-DLK1 (1:200); magnification, $\mathrm{x} 200$ and 400] in cardiac tissues from 25 patients with TOF and five controls. The $\mathrm{x} 400$ image is the enlarged part, which is inside the rectangle in the $\mathrm{x} 200$ image. (B) Semi-quantitative analysis of DLK1 expression. ${ }^{* * *} \mathrm{P}<0.001$. DLK1, $\delta$ like non-canonical Notch ligand 1 ; TOF, Tetralogy of Fallot.

protein transfer. The membrane was blocked with $5 \%$ skimmed milk $(0.05 \mathrm{~g} / \mathrm{ml})$ for $\sim 1 \mathrm{~h}$ at room temperature, incubated with the primary antibody against ETS1 (1:1,000; cat. no. D8O8A; Cell Signaling Technology, Inc.) at $4^{\circ} \mathrm{C}$ overnight, and then incubated with a HRP-conjugated anti-rabbit secondary antibody (1:5,000; cat. no. M21002; Abmart Pharmaceutical Technology Co., Ltd.) for $\sim 2 \mathrm{~h}$ at room temperature. Finally, the blots were visualized using enhanced chemiluminescence reagents (Thermo Fisher Scientific, Inc.).

Chromatin immunoprecipitation-quantitative (ChIP-q) $P C R$. 293 cells were seeded in two $10 \mathrm{~cm}$ dishes at a density of $2 \times 10^{6}$ cells/well. After $24 \mathrm{~h}$, the experimental group was treated with $40 \mu \mathrm{M}$ 5-Aza-2'-deoxycytidine (Sigma Aldrich; Merck $\mathrm{KGaA}$ ), and the control group was treated with the same volume of DMSO. The treatment of both groups lasted for $\sim 48 \mathrm{~h}$ at $37^{\circ} \mathrm{C}$ under $5 \% \mathrm{CO}_{2}$. The ChIP assay was performed using EZ-Magna ChIP ${ }^{\text {Tм }}$ A/G Chromatin Immunoprecipitation kit (MilliporeSigma) according to the manufacturer's instructions. Briefly, cells were incubated with $225 \mu 137 \%$ formaldehyde (final concentration, 1\%) for $\sim 10 \mathrm{~min}$ at room temperature, then $1 \mathrm{ml} 10 \mathrm{X}$ glycine was added to stop crosslinking for $\sim 5 \mathrm{~min}$ at room temperature and collected with cold 1X PBS. After resuspension in $500 \mu 1$ SDS lysis buffer, samples were then sonicated by ultrasonic disruptor (Diagenode Bioruptor; intensity 5, $30 \mathrm{sec}$ on and $30 \mathrm{sec}$ off, 25 cycles) and centrifuged at $10,000 \times \mathrm{g}$ at $4^{\circ} \mathrm{C}$ for $10 \mathrm{~min}$ to remove insoluble material. Sheared chromatin $(50 \mu \mathrm{l})$ was incubated with $450 \mu \mathrm{l}$ ChIP dilution buffer and antibody overnight at $4^{\circ} \mathrm{C}$ with rotation. Antibodies used for ChIP included: Anti-ETS1 antibody Chip grade (1:50; cat. no. D8O8A; Cell Signaling Technology, Inc.) and anti-rabbit IgG Chip grade (5 $\mu$ g; cat. no. 2729S; Cell Signaling Technology, Inc.). The antibody of IgG was used as the negative control. The next day, the magnetic beads were washed with low salt wash buffer, high salt wash buffer, $\mathrm{LiCl}$ wash buffer and TE buffer at room temperature. Each sample was incubated with $100 \mu \mathrm{l} \mathrm{ChIP}$ elution buffer at $62^{\circ} \mathrm{C}$ for $2 \mathrm{~h}$ with shaking, at $95^{\circ} \mathrm{C}$ for $10 \mathrm{~min}$ and then cooled down to room temperature. Beads were then separated using magnets, and the supernatant was purified using FastPure Gel DNA Extraction Mini Kit (cat. no. DC301; Vazyme Biotech Co., Ltd.) to acquire DNA fragments. The enriched DNA fragments were quantified via qPCR using TB Green ${ }^{\circledR}$ Premix Ex Taq ${ }^{\mathrm{TM}}$ (cat. no. RR420A; Takara Bio, Inc.). Thermocycling conditions were as follows: $95^{\circ} \mathrm{C}$ for $30 \mathrm{sec}$; 40 cycles of $95^{\circ} \mathrm{C}$ for $5 \mathrm{sec}$ and $60^{\circ} \mathrm{C}$ for $20 \mathrm{sec}$; and $95^{\circ} \mathrm{C}$ for $15 \mathrm{sec}$ and $60^{\circ} \mathrm{C}$ for $60 \mathrm{sec}$. Three independent qPCRs were performed. The primers used for ChIP-qPCR were as follows: ChIP-qPCR-F, 5'-TTTGTGTTTCAGCGCGGCTAAG-3' and ChIP-qPCR-R, 5'-TTACCCAACCATGGGCATCCTCC-3'. The obtained results were expressed as \% input (18).

Statistical analysis. All data are presented as the median (interquartile range) of three independent experiments. Data were analyzed using GraphPad Prism software (version 7.0, GraphPad Software, Inc.). The differences between DLK1 expression and the methylation status of the DLK1 gene promoter in the two groups were determined with a Mann-Whitney test. Pearson's correlation was used to test the correlation between methylation levels and protein expression. The differences in luciferase activity and the enrichment of the amplified fragments between multiple groups were tested by one-way ANOVA, followed by the LSD post hoc test. $\mathrm{P}<0.05$ was considered to indicate a statistically significant difference.

\section{Results}

Expression of DLK1 protein in the RVOT tissues of patients with TOF and controls. To determine the clinical relevance of DLK1 in the development of TOF, immunohistochemistry was used to detect the expression level of DLK1 protein in the RVOT tissues of 25 patients with TOF and five controls. As shown in Fig. 1A, the intensity of positive yellow-brown immunohistochemical staining of DLK1 protein in the RVOT tissues was markedly weaker in patients with TOF than that in controls (Fig. 1A). The further semi-quantitative analysis showed that the expression of DLK1 protein in the patients with TOF was significantly lower than that in the controls $(\mathrm{P}<0.001$; Fig. 1B). 
A

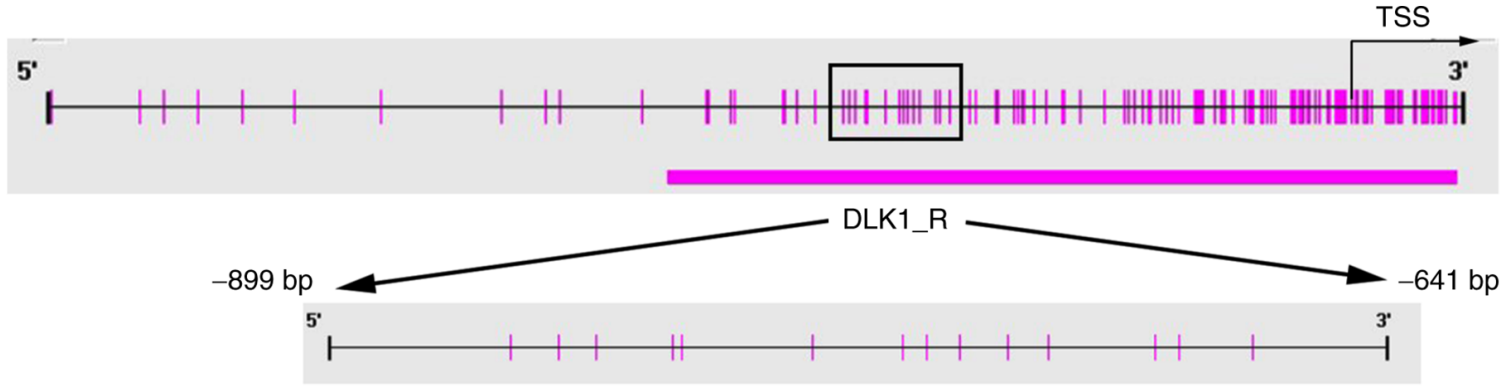

B

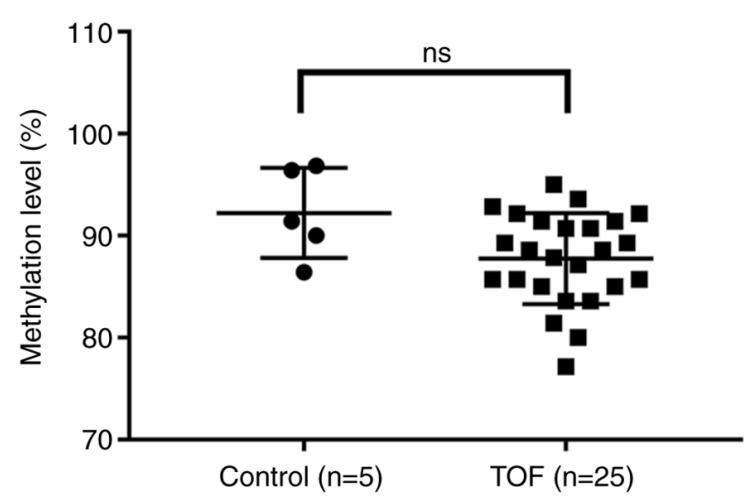

C

- Control $(n=5)$

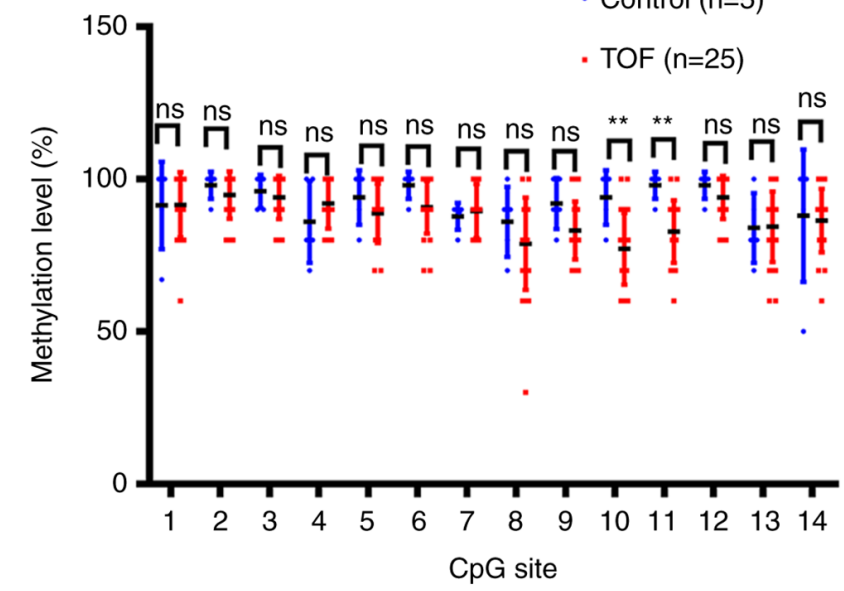

Figure 2. Methylation status of the DLK1 promoter region in cardiac tissues. (A) The presentation of DLK1_R (-899 bp/-641 bp) in the DLK1 promoter region . (B) The integral methylation status of DLK1_R (-899 bp/-641 bp) in the 25 patients with TOF and five controls. (C) The methylation level of each CpG site in the DLK1_R region in patients with TOF $(n=25)$ and healthy controls $(n=5) .{ }^{* *} \mathrm{P}<0.01$. DLK1, $\delta$ like non-canonical Notch ligand 1; TOF, Tetralogy of Fallot; ns, no significance; TSS, transcription start site.
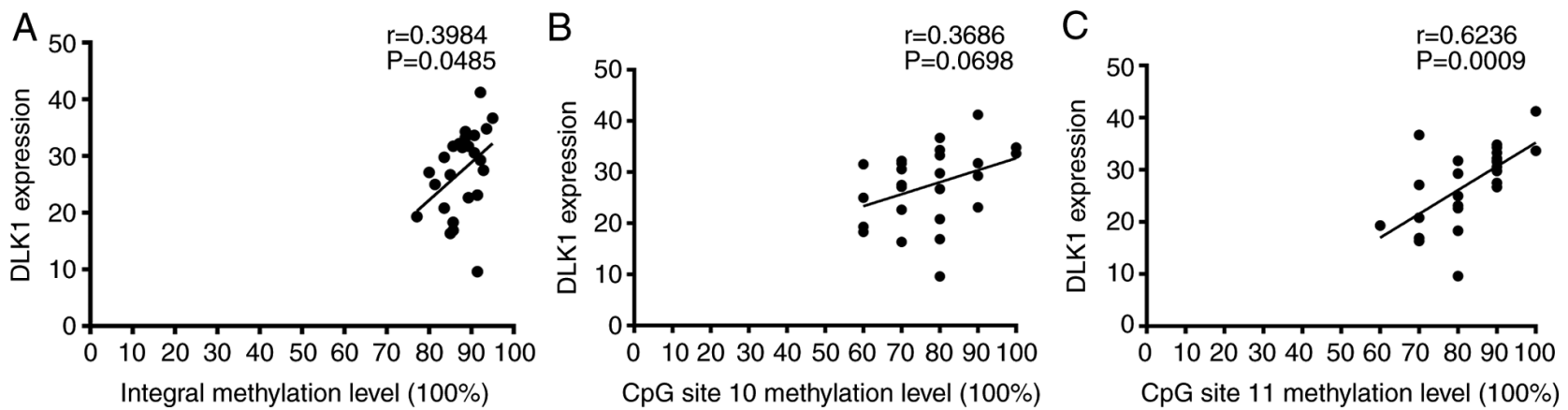

Figure 3. Correlation between methylation level and DLK1 expression in 25 patients with TOF. (A) Correlation between integral methylation level of the DLK1_R region and DLK1 expression in 25 patients with TOF. (B) Correlation between the CpG site 10 methylation level and DLK1 expression in 25 patients with TOF. (C) Correlation between the CpG site 11 methylation level and DLK1 expression in 25 patients with TOF. DLK1, $\delta$ like non-canonical Notch ligand 1; TOF, Tetralogy of Fallot.

Methylation status analysis for DLK1 gene in patients with TOF and controls. BSP was performed to measure the methylation level of the DLK1 gene promoter. The amplicon (DLK1_R, -899 to -641 bp) from the DLK1 gene promoter was analyzed in 25 patients with TOF and five age-matched controls (Fig. 2A). The results underwent quality control to remove unreliable methylation data.

The integral methylation level of DLK1_R was not significantly different in 25 TOF patients with a median of $88.57 \%$ (IQR, 85-91.43\%), compared with the five controls with a median of 91.43\% (IQR, 88.21-96.64\%) ( $\mathrm{P}=0.0716$; Fig. 2B).
Of note, the subsequent analysis of the methylation status of each CpG site in the DLK1_R region revealed that the methylation level of $\mathrm{CpG}$ site 10 and $\mathrm{CpG}$ site 11 was significantly lower in the TOF group than in the control group $(\mathrm{P}<0.01$; Fig. $2 \mathrm{C})$.

Pearson's correlation analysis was used to determine whether DLK1_R methylation level was related to the expression level of DLK1 protein. There was a moderate positive correlation between the integral methylation status of DLK1_R and DLK1 expression in 25 patients with TOF ( $r=0.3984, \mathrm{P}=0.0485$; Fig. 3A). DLK1 protein expression was not significantly correlated with the methylation level of $\mathrm{CpG}$ site 10 ( $\mathrm{r}=0.3686, \mathrm{P}=0.0698$; Fig. 3B). 
A

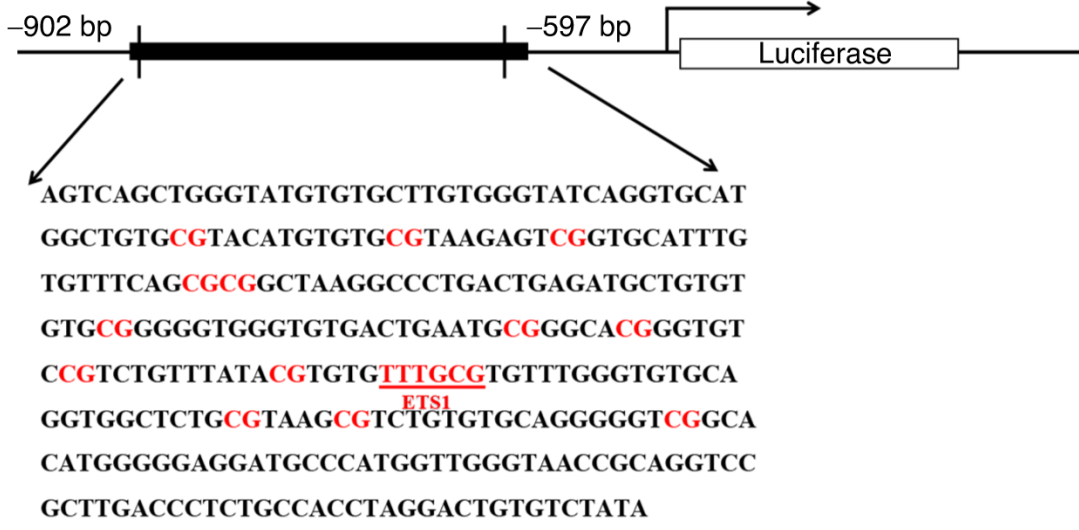

B

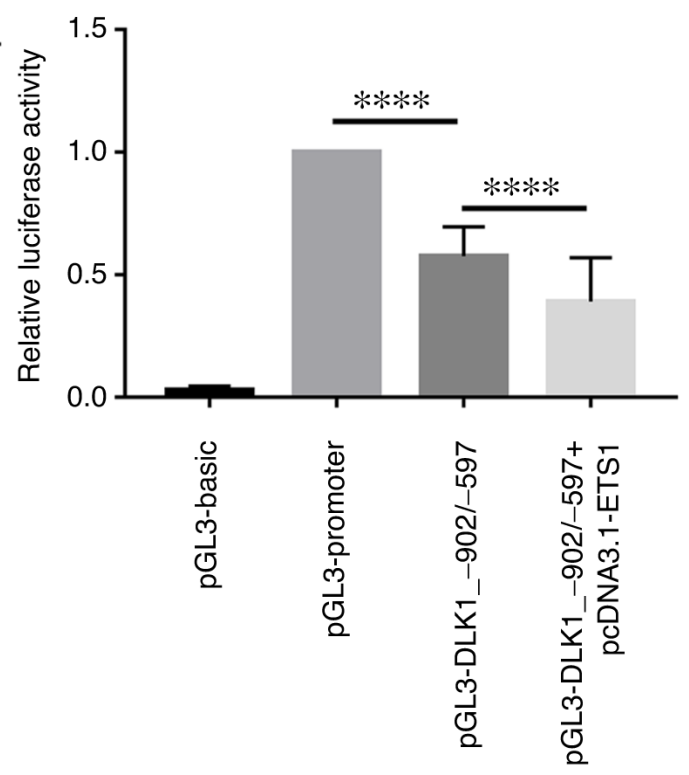

Figure 4. Regulation of ETS1 on the DLK1_-902/-597 region. (A) The sequence of the DLK1_-902/-597 region and the binding sites of ETS1. (B) Luciferase activity of pGL3-DLK1_-902/-597 co-transfected with ETS1 in the 293 cell line. ${ }^{* * * *} \mathrm{P}<0.0001$. DLK1, $\delta$ like non-canonical Notch ligand 1; ETS1, ETS proto-oncogene 1 .

However, a moderate positive correlation was also observed between DLK1 expression and the methylation status of $\mathrm{CpG}$ site 11 in patients with TOF ( $\mathrm{r}=0.6236, \mathrm{P}=0.0009$; Fig. $3 \mathrm{C})$.

ETS1 transcription factor binds to the promoter region of DLK1 and inhibits gene transcription activity. To explore the influence of the DLK1_R region on gene transcription activity, a dual-luciferase assay was performed using 293 cells. The pGL3-DLK1_-902/-597 plasmid was constructed by placing the DLK1_-902/-597 fragment containing the DLK1_R region (-899 bp to -641 bp) under pGL3-promoter (Fig. 4A). As shown in Fig. 4B, the luciferase activity in the case of the pGL3-DLK1_-902/-597 vector was significantly lower than in the case of the plasmid harboring the pGL3-promoter and lacking the DLK1_-902/-597 fragment, which indicated that the examined DLK1_-902/-597 region can inhibit gene transcription activity $(\mathrm{P}<0.0001)$.

An ETS1-binding sequence (-728 bp to -723 bp) containing the $\mathrm{CpG}$ site 11 (-724 bp) was predicted to be located in the DLK1_R region of the DLK1 promoter using the JASPAR database (Fig. 4A). To verify this prediction, pcDNA3.1-ETS1 was co-transfected with pGL3-DLK1_-902/-597 into 293 cells and the luciferase activity assay was performed. The luciferase activity in the case of pGL3-DLK1_-902/-597 + pcDNA3.1-ETS1 was significantly reduced compared with the pGL3-DLK1_-902/-597 transfection alone ( $\mathrm{P}<0.0001$; Fig. 4B).

These findings suggested that the ETS1-transcription factor could bind to the promoter region of DLK1 and inhibit the transcriptional activity of DLK1.

Validation of binding of ETS1 to the DLK1 promoter in vitro. To determine whether the transcription factor ETS1 could directly bind to the $\mathrm{CpG}$ site 11 and whether the methylation of DLK1_R could affect ETS1 binding affinity, EMSAs were performed using the overexpressed ETS1 protein (Fig. 5A) and oligonucleotide probes that contained ETS1 binding sequences. As shown in Fig. 5B (lane 2), a DNA-protein complex was formed when the biotin-labeled probes were incubated with ETS1. When unlabeled probes were added for competition, the ETS1 binding was inhibited, which resulted in a lighter band (lane 3). However, the bands in lane 4 and lane 5, where the mutation probes and methylated probes were added, were even lighter than that in lane 3. The subsequent Shift-western blotting showed that the delayed band detected in EMSA was the result of ETS1 protein binding (Fig. 5B). These findings demonstrated that the ETS1 transcription factor could bind to the promoter of DLK1 and that the methylation status of DLK1_R was able to influence ETS1 binding affinity.

Validation of binding of ETS1 to the DLK1 promoter in vivo. ChIP DNA samples and input DNA samples were quantified via qPCR. The fragments containing the predicted ETS1 transcription factor binding sequences were significantly enriched in the anti-ETS1 group, when compared with the anti-IgG group $(\mathrm{P}<0.001$; Fig. 5C). When 293 cells were treated with DNA-demethylating agent 5-AZA-dC, the enrichment of the fragment was higher than that in the control group without 5-AZA-dC ( $\mathrm{P}<0.01$; Fig. 5C).

\section{Discussion}

The Notch pathway is a highly conserved cell signaling pathway required for cell fate specification and tissue patterning in the embryo (19). A consistent set of data in animal models and 

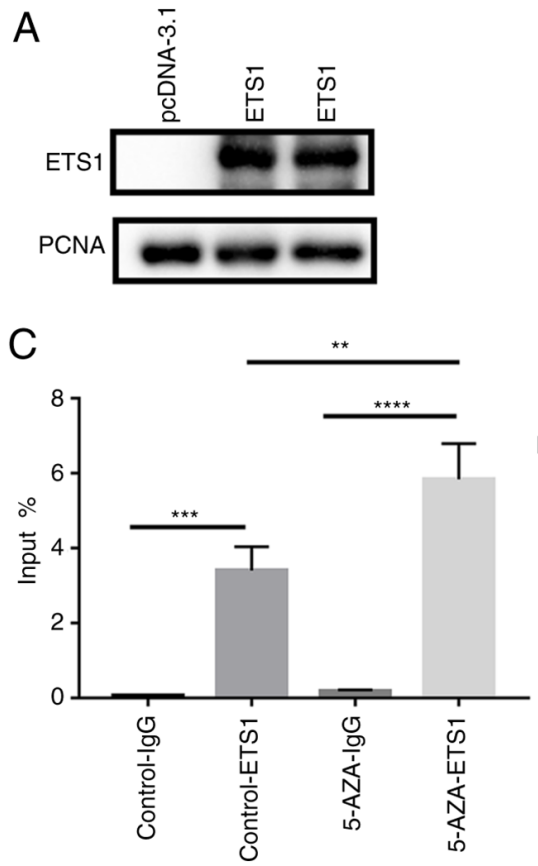

B

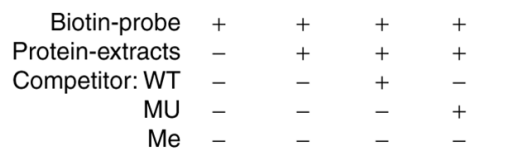

$+$

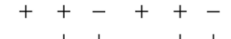

$-++-++$
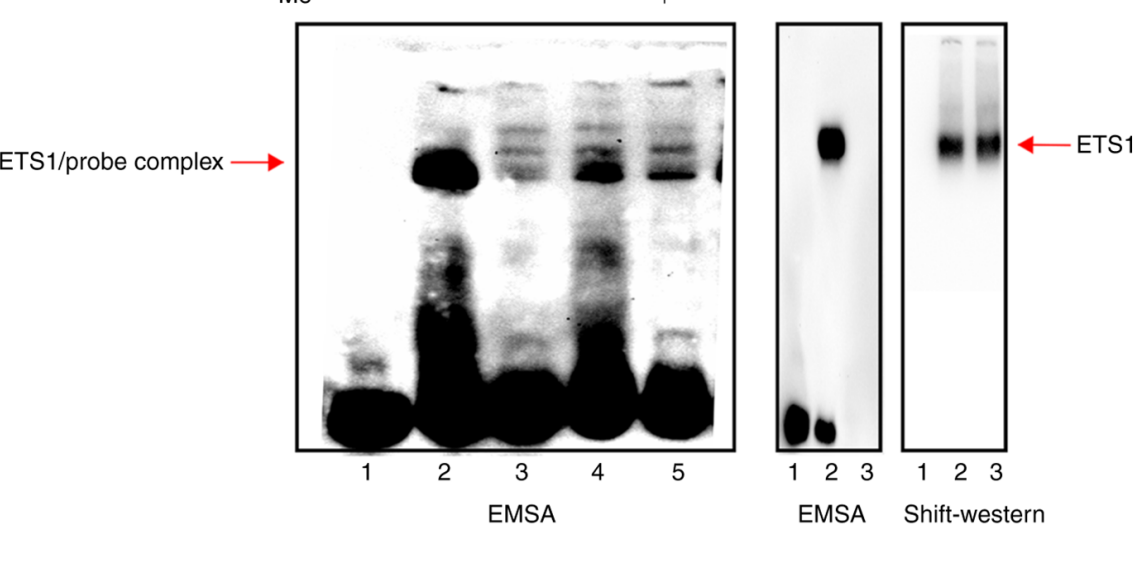

Figure 5. Binding of ETS1 to the DLK1 promoter. (A) Overexpression of ETS1 was verified via western blotting. (B) EMSA: i) Lane 1, biotin-labeled probes alone; ii) lane 2, biotin-labeled probes were incubated with ETS1 nuclear protein; iii) lane 3, unlabeled probes as competitors were added based on lane 2 ; iv) lane 4 , mutation probes as competitors were added based on lane 2; and v) lane 5 , methylated probes as competitors were added based on lane 2. Shift-western blotting confirmed ETS1 binding. (C) Chromatin immunoprecipitation assay was performed with anti-ETS1 using 293 cells treated with or without 5-aza. Quantitative PCR was used to verify the enrichment of DLK1_R. ${ }^{* *} \mathrm{P}<0.01,{ }^{* * *} \mathrm{P}<0.001,{ }^{* * * *} \mathrm{P}<0.0001$. DLK1, $\delta$ like non-canonical Notch ligand 1 ; ETS1, ETS proto-oncogene 1; EMSA, electrophoretic mobility shift assay; 5-AZA, 5-azacytidine; WT, wild-type; MU, mutant; Me, methylated; PCNA, proliferating cell nuclear antigen.

humans has demonstrated that the Notch signaling pathway plays an important role in cardiogenesis (20-22). DLK1, one of the Notch ligands, is commonly expressed during fetal development and highly expressed in cardiac development (9). In the current study, by using immunohistochemistry, it was found that DLK1 protein expression in RVOT tissues of patients with TOF was significantly lower than that in controls. Considering the very low frequency of DLK1 in patients with TOF and the importance of epigenetics on the pathogenesis of TOF, it was deduced that the epigenetic changes may be closely associated with the abnormal expression of the DLK1 gene.

Several studies have demonstrated that epigenetic mechanisms play an important role in cardiac development by altering the expression of cardiac-related genes $(23,24)$. One of the epigenetic modifications regulating gene transcription is DNA methylation status (25). Aberrant methylation of promoter $\mathrm{CpG}$ islands is known to contribute to the phenotypic expression of CHDs, including TOF and VSD (3). Our previous studies identified that the altered expression of some genes involved in cardiac development, such as Notch4 and COUP transcription factor 2 in TOF, may be associated with the abnormal methylation of their promoters $(26,27)$. In the current study, no significant difference was observed in the integral methylation level of DLK1 promoter between the patients with TOF and controls. However, previous studies have demonstrated that the methylation of certain $\mathrm{CpG}$ sites can affect gene expression $(26,28,29)$. Therefore, the methylation status of each CpG site in the DLK1_R region was analyzed in the present study. $\mathrm{CpG}$ site 10 and $\mathrm{CpG}$ site 11 had lower methylation levels in patients with TOF compared with controls. Moreover, a significant positive correlation between the DLK1 methylation level and DLK1 expression in patients with TOF could be observed in the $\mathrm{CpG}$ site 11 . It was deduced that the hypomethylation level of the $\mathrm{CpG}$ site 11 may be associated with lower expression of DLK1 in patients with TOF compared with controls.

The present findings differ from previous studies that have reported a repressive role of hypermethylation in gene expression, which acts by preventing transcription factors from binding to CGI promoters $(30,31)$. However, several publications have reported similar findings that promoter hypermethylation can facilitate gene transcription, mainly in tumor occurrence, development and metastasis (32-34). The most commonly hypothesized mechanism is that the increased methylation of the promoter can prevent the binding of a repressive transcription factor, which facilitates active gene transcription (35). To verify this hypothesis, the JASPAR database was used in the current study and it was found that ETS1 is a potential transcription factor that can bind to the DLK1_R region containing the $\mathrm{CpG}$ site 11. ETS1, as a member of the ETS family required for normal vascular development, can mediate early cardiomyocyte development in the embryo $(36,37)$. The current research determined that ETS1 could bind to the DLK1 promoter and inhibit gene activity. This finding was consistent with the aforementioned previous studies.

To further investigate whether the methylation status of the DLK1_R region could affect the binding of ETS1, EMSA Shift-western blotting and ChIP-qPCR were performed. EMSA demonstrated that ETS1 could directly bind to the promoter of DLK1, and its binding could be blocked by 
methylation. Moreover, Shift-western blotting confirmed that the band detected as a result of EMSA was indeed the ETS1 protein. Furthermore, ChIP-qPCR was performed and it was determined that DNA-demethylating agent 5-AZA-dC increased the enrichment of the DLK1_R fragment in ETS1. Taken together, these findings suggested that ETS1 could bind to the DLK1 promoter, with its affinity affected by the methylation status of the DLK1 promoter. This dependence proved to influence DLK1 protein expression. Although these results clarified that ETS1 could regulate gene expression by directly binding to the DLK1 promoter, the specific mechanism of demethylation and repression needs further exploration.

There are several limitations of the present study. The first limitation of this study was the insufficient number of samples due to the difficulty in obtaining cardiac tissues, especially from healthy controls. In addition, it could not be determined whether TOF caused abnormal methylation of DLK1 or whether this abnormality contributed to TOF. Therefore, the aforementioned issues need to be further explored in animal models or a prospective cohort. The second limitation was that 293 cells were used rather than cardiomyocytes, 293 cells were used in order to improve transfection efficiency as the transfection efficiency using cardiomyocytes was very low and not suitable for experiments.

In summary, this study demonstrated that the hypomethylation of specific sites in the DLK1_R region may influence DLK1 gene expression in patients with TOF. ETS1 proved to be a repressive transcription factor binding to the DLK1 promoter. Therefore, hypomethylation of $\mathrm{CpG}$ site 10 and CpG site 11 may increase the binding affinity of ETS1 and reduce DLK1 expression levels in patients with TOF. Given the aforementioned information, this study has the potential to provide novel epigenetic insights into the pathogenesis of TOF and contribute to further research on this subject.

\section{Acknowledgements}

Not applicable.

\section{Funding}

This study was funded by the National Key Research and Development Program of China (grant no. 2021YFC2701000), the National Natural Science Foundation of China (grant nos. 81873482 and 81873483), Shanghai Basic Research Project of Science and Technology Innovation Action Plan (grant no. 20JC1418300), Youth Program of National Natural Science Foundation of China (grant no. 81800282), CAMS Innovation Fund for Medical Sciences (grant no. 2019-I2M-5-002) and the Shanghai Sailing Program (grant no. 18YF1402600).

\section{Availability of data and materials}

The datasets used and/or analyzed during the current study are available from the corresponding author on reasonable request.

\section{Authors' contributions}

WS and GH made major contributions to the design of this study. GT, LH and RG performed the experiments and wrote the manuscript. JS, WC and YQ collected the samples and patient information, and analyzed the clinical data. ZZ, XM and WY participated in analyzing the experimental data. ZX and MS prepared the tables and figures, and made contributions to the interpretation of data. GT and WS confirm the authenticity of all the raw data. All authors have read and approved the final manuscript.

\section{Ethics approval and consent to participate}

The Ethics Committee of the Children's Hospital of Fudan University [Shanghai, China; approval no. 2015(26)] and Soochow University (Suzhou, China) approved the collection of cardiac tissues. Written informed consent of all study participants was obtained from their parents or relatives.

\section{Patient consent for publication}

Not applicable.

\section{Competing interests}

The authors declare that they have no competing interests.

\section{References}

1. Apitz C, Webb GD and Redington AN: Tetralogy of Fallot. Lancet 374: 1462-1471, 2009.

2. Page DJ, Miossec MJ, Williams SG, Monaghan RM, Fotiou E, Cordell HJ, Sutcliffe L, Topf A, Bourgey M, Bourque G, et al: Whole exome sequencing reveals the major genetic contributors to Nonsyndromic Tetralogy of Fallot. Circ Res 124: 553-563, 2019.

3. Grunert M, Dorn C, Cui H, Dunkel I, Schulz K, Schoenhals S, Sun W, Berger F, Chen W and Sperling SR: Comparative DNA methylation and gene expression analysis identifies novel genes for structural congenital heart diseases. Cardiovasc Res 112: 464-477, 2016.

4. Finn J, Sottoriva K, Pajcini KV, Kitajewski JK, Chen C, Zhang W, Malik AB and Liu Y: Dlk1-Mediated Temporal Regulation of Notch Signaling is required for differentiation of Alveolar Type II to Type I cells during repair. Cell Rep 26: 2942-2954. e5, 2019.

5. de la Pompa JL and Epstein JA: Coordinating tissue interactions: Notch signaling in cardiac development and disease. Dev Cell 22: 244-254, 2012.

6. McCright B, Lozier J and Gridley T: A mouse model of Alagille syndrome: Notch2 as a genetic modifier of Jag1 haploinsufficiency. Development 129: 1075-1082. 2002.

7. Digilio MC, Luca AD, Lepri F, Guida V, Ferese R, Dentici ML, Angioni A, Marino B and Dallapiccola B: JAG1 mutation in a patient with deletion 22q11.2 syndrome and tetralogy of Fallot. Am J Med Genet A 161A: 3133-3136, 2013.

8. Garg V: Notch signaling in aortic valve development and disease. In: Etiology and Morphogenesis of Congenital Heart Disease: From Gene Function and Cellular Interaction to Morphology. Nakanishi T, Markwald RR, Baldwin HS, Keller BB, Srivastava D and Yamagishi H (eds). Springer, Tokyo, pp 371-376, 2016.

9. Huang CC, Kuo HM, Wu PC, Cheng SH, Chang TT, Chang YC, Kung ML, Wu DC, Chuang JH and Tai MH: Soluble delta-like 1 homolog (DLK1) stimulates angiogenesis through Notch1/Akt/eNOS signaling in endothelial cells. Angiogenesis 21: 299-312, 2018.

10. Shamis Y, Cullen DE, Liu L, Yang G, Ng SF, Xiao L, Bell FT, Ray C, Takikawa S, Moskowitz IP, et al: Maternal and zygotic Zfp57 modulate NOTCH signaling in cardiac development. Proc Natl Acad Sci USA 112: E2020-2029, 2015.

11. Rodriguez P, Sassi Y, Troncone L, Benard L, Ishikawa K, Gordon RE, Lamas S, Laborda J, Hajjar RJ and Lebeche D: Deletion of delta-like 1 homologue accelerates fibroblast-myofibroblast differentiation and induces myocardial fibrosis. Eur Heart J 40: 967-978, 2019. 
12. Zhu H, Wang $\mathrm{G}$ and Qian J: Transcription factors as readers and effectors of DNA methylation. Nat Rev Genet 17: 551-565, 2016.

13. Moore-Morris T, van Vliet PP, Andelfinger G and Puceat M: Role of epigenetics in cardiac development and congenital diseases. Physiol Rev 98: 2453-2475, 2018.

14. Serra-Juhé C, Cuscó I, Homs A, Flores R, Torán N and Pérez-Jurado LA: DNA methylation abnormalities in congenital heart disease. Epigenetics 10: 167-177, 2015.

15. Cao J, Wu Q, Huang Y, Wang L, Su Z and Ye H: The role of DNA methylation in syndromic and non-syndromic congenital heart disease. Clin Epigenetics 13: 93, 2021.

16. Sheng W, Qian Y, Wang H, Ma X, Zhang P, Diao L, An Q, Chen L, Ma D and Huang G: DNA methylation status of NKX2-5, GATA4 and HAND1 in patients with tetralogy of fallot. BMC Med Genomics 6: 46, 2013

17. Fornes O, Castro-Mondragon JA, Khan A, van der Lee R, Zhang X, Richmond PA, Modi BP, Correard S, Gheorghe M, Baranasic D, et al: JASPAR 2020: update of the open-access database of transcription factor binding profiles. Nucleic Acids Res 48 (D1): D87-D92, 2020.

18. Lacazette E: A laboratory practical illustrating the use of the ChIP-qPCR method in a robust model: Estrogen receptor alpha immunoprecipitation using Mcf-7 culture cells. Biochem Mol Biol Educ 45: 152-160, 2017.

19. Kuhnert F, Kirshner JR and Thurston G: Dll4-Notch signaling as a therapeutic target in tumor angiogenesis. Vasc Cell 3: 20, 2011.

20. MacGrogan D, Münch J and de la Pompa JL: Notch and interacting signalling pathways in cardiac development, disease, and regeneration. Nat Rev Cardiol 15: 685-704, 2018.

21. Luxan G, D'Amato G, MacGrogan D and de la Pompa JL: Endocardial Notch signaling in cardiac development and disease. Circ Res 118: e1-e18, 2016.

22. Chen H, Zhang W, Sun X, Yoshimoto M, Chen Z, Zhu W, Liu J, Shen Y, Yong W, Li D, et al: Fkbpla controls ventricular myocardium trabeculation and compaction by regulating endocardial Notch1 activity. Development 140: 1946-1957, 2013.

23. van Weerd JH, Koshiba-Takeuchi K, Kwon C and Takeuchi JK: Epigenetic factors and cardiac development. Cardiovasc Res 91: 203-211, 2011

24. Wang Z, Zhai W, Richardson JA, Olson EN, Meneses JJ, Firpo MT, Kang C, Skarnes WC and Tjian R: Polybromo protein BAF180 functions in mammalian cardiac chamber maturation. Genes Dev 18: 3106-3116, 2004.

25. Jones PA: Functions of DNA methylation: Islands, start sites, gene bodies and beyond. Nat Rev Genet 13: 484-492, 2012.
26. Zhu Y, Ye M, Xu H, Gu R, Ma X, Chen M, Li X, Sheng W and Huang G: Methylation status of $\mathrm{CpG}$ sites in the NOTCH4 promoter region regulates NOTCH4 expression in patients with tetralogy of Fallot. Mol Med Rep 22: 4412-4422, 2020.

27. Xiaodi L, Ming Y, Hongfei X, Yanjie Z, Ruoyi G, Ma X, Wei S and Guoying $\mathrm{H}$ : DNA methylation at $\mathrm{CpG}$ island shore and RXR $\alpha$ regulate NR2F2 in heart tissues of tetralogy of Fallot patients. Biochem Biophys Res Commun 529: 1209-1215, 2020.

28. Vohra M, Adhikari P, Souza SC, Nagri SK, Umakanth S, Satyamoorthy K and Rai PS: CpG-SNP site methylation regulates allele-specific expression of MTHFD1 gene in type 2 diabetes. Lab Invest 100: 1090-1101, 2020.

29. Dayeh TA, Olsson AH, Volkov P, Almgren P, Ronn T and Ling C: Identification of CpG-SNPs associated with type 2 diabetes and differential DNA methylation in human pancreatic islets. Diabetologia 56: 1036-1046, 2013.

30. Bogdanović $\mathrm{O}$ and Lister R: DNA methylation and the preservation of cell identity. Curr Opin Genet Dev 46: 9-14, 2017.

31. Greenberg MVC and Bourc'his D: The diverse roles of DNA methylation in mammalian development and disease. Nat Rev Mol Cell Biol 20: 590-607, 2019.

32. Bahar Halpern K, Vana T and Walker MD: Paradoxical role of DNA methylation in activation of FoxA2 gene expression during endoderm development. J Biol Chem 289: 23882-23892, 2014.

33. Nabilsi NH, Broaddus RR and Loose DS: DNA methylation inhibits p53-mediated survivin repression. Oncogene 28: 2046-2050, 2009.

34. Jia N, Wang J, Li Q, Tao X, Chang K, Hua K, Yu Y, Wong KK and Feng W: DNA methylation promotes paired box 2 expression via myeloid zinc finger 1 in endometrial cancer. Oncotarget 7: 84785-84797, 2016.

35. Smith J, Sen S, Weeks RJ, Eccles MR and Chatterjee A: Promoter DNA Hypermethylation and Paradoxical Gene Activation. Trends Cancer 6: 392-406, 2020.

36. Ruan H, Liao Y, Ren Z, Mao L, Yao F, Yu P, Ye Y, Zhang Z, Li S, $\mathrm{Xu} \mathrm{H}$, et al: Single-cell reconstruction of differentiation trajectory reveals a critical role of ETS1 in human cardiac lineage commitment. BMC Biol 17: 89, 2019.

37. Nie S and Bronner ME: Dual developmental role of transcriptional regulator Ets1 in Xenopus cardiac neural crest vs. heart mesoderm. Cardiovasc Res 106: 67-75, 2015.

(i) $\ominus$ This work is licensed under a Creative Commons Attribution-NonCommercial-NoDerivatives 4.0 International (CC BY-NC-ND 4.0) License. 